\title{
Verbesserung der Lebensqualität schon bei Beginn motorischer Komplikationen?
}

Fragestellung: Ist die Behandlung des M. Parkinson durch tiefe Hirnstimulation bereits nach dem ersten Auftreten motorischer Fluktuationen und Dyskinesien der alleinigen medikamentösen Therapie überlegen?

Hintergrund: Nach der sogenannten Honeymoon-Phase mit gutem Ansprechen auf die medikamentöse Therapie des M. Parkinson können im Verlauf Wirkfluktuationen und Dyskinesien als Nebenwirkung der L-Dopa-Therapie auftreten. Die tiefe Hirnstimulation des N. subthalamicus (STN) ist eine erfolgreiche Option bei fortgeschrittenem M. Parkinson, wenn diese motorischen Komplikationen trotz Ausschöpfen medikamentöser Maßnahmen weiterhin bestehen. Die durchschnittliche Krankheitsdauer bis zur Operation beträgt bisher zwölf Jahre. Unklar ist, ob sich die Lebensqualität durch einen frühzeitigen $\mathrm{Be}$ -

Schuepbach WMM, Rau J, Knudsen J et al; EARLYSTIM Study Group. Neurostimulation for Parkinson's Disease with Early Motor Complications. N Engl J Med 2013; 368: 610-22 ginn der tiefen Hirnstimulation kurz nach Auftreten von Fluktuationen oder Dyskinesien bessern lässt.

Patienten und Methodik: Insgesamt 251 Patienten (mittleres Alter 52 Jahre) mit einer mittleren Krankheitsdauer von sieben Jahren wurden entweder nur medikamentös behandelt oder ergänzend mit tiefer Hirnstimulation. Voraussetzung war, dass die motorischen Komplikationen seit höchstens drei Jahren bestanden. Primärer Endpunkt war die Verbesserung der Lebensqualität, gemessen mithilfe des Parkinson's Disease Questionnaire (PDQ-39). Sekundäre Endpunkte waren motorische Beeinträchtigungen (UPDRS-III-Skala), Aktivitäten des täglichen Lebens (UPDRS-II-Skala) und Beweglichkeit.

Ergebnisse: Nach zweijähriger Beobachtungszeit hatten die Patienten mit Neurostimulation einen um acht Punkte besseren Wert im PDQ-39 (Score von 0-100) als die rein medikamentös behandelten $(p=0,002)$. Auch bei den sekundären Endpunkten motorische Beeinträchtigungen und Aktivitäten des täglichen Lebens $(\mathrm{p}<0,001)$ sowie Zeiten guter Beweglichkeit $(\mathrm{p}=0,01)$ schnitten die neurostimulierten Patienten besser ab. Die einzigen Todesfälle traten nach Suiziden auf (zwei in der Stimulationsgruppe, einer in der Kontrollgruppe).

Schlussfolgerungen: Die tiefe Hirnstimulation des STN ist der alleinigen medikamentösen Therapie beim M. Parkinson bereits in der frühen Phase motorischer Komplikationen überlegen.

\section{Mögliche Option bei beginnenden Fluktuationen oder Dyskinesien}

Diese deutsch-französische Studie wurde mit hohem Aufwand und großer Sorgfalt durchgeführt. Das erstaunliche Ergebnis ist, dass in einer Phase, in der noch eine Vielzahl medikamentöser Optionen bestehen, durch die tiefe Hirnstimulation die motorischen Beeinträchtigungen vermindert werden und die Lebensqualität erheblich verbessert wird. Trotz der eindeutigen Ergebnisse der Studie müssen aber noch einige Fragen beantwortet werden, bevor die tiefe Hirnstimulation in einem frühen Krankheitsstadium allgemein empfohlen werden kann. Weitergehende Fragen zu frontalen Hirnfunktionsstörungen infolge der tiefen Hirnstimulation, Auswirkungen auf die Identität der Patienten und das Lebensumfeld konnten in der Earlystim-Studie nicht geklärt werden. Das erneut dokumentierte Suizidrisiko bei Patienten nach tiefer Hirnstimulation hängt ganz erheblich von der Sorgfalt der Nachbetreuung sowie der psychiatrischen Erfahrung des Arztes ab und könnte im Behandlungsalltag höher sein als unter den Studienbedingungen. Die Frage, ob der frühe Behandlungsbeginn einem späteren über die gesamte Krankheitsdauer überlegen ist, wird möglicherweise ein Follow-up mit einem Beobachtungszeitraum von einigen weiteren Jahren beantworten. Es wäre erfreulich gewesen, wenn diese wichtige Studie ohne die finan- zielle Unterstützung des Herstellers der Stimulationssysteme ausgekommen wäre.

Die Studie rechtfertigt in Einzelfällen eine frühere Behandlung, allerdings erst bei Wirkungsfluktuationen oder Dyskinesien. Eine grundsätzliche Empfehlung zur Operation in dieser Phase des M. Parkinson kann noch nicht abgeleitet werden. Es bedarf weiterhin einer individuellen Indikationsstellung, die erfahrenen Zentren vorbehalten bleiben sollte. Die Nachsorge erfordert engmaschige Kontrollen und Erfahrung in der Beurteilung psychiatrischer Störungen, insbesondere der Suizidalität.

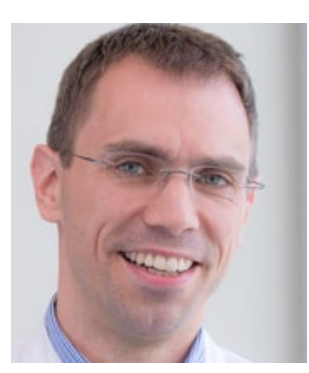

PD Dr. med. Markus Frings, Essen

Oberarzt der Klinik für Neurologie, Universitätsklinikum Essen E-Mail: markus.frings@uni-due.de 